\title{
Economical and Green Acetaldehyde to Glyoxal Electroorganic Conversion: a Theoretical Study
}

\author{
Volodymyr V. Tkach ${ }^{1,2, *}$ (D) , Marta V. Kushnir ${ }^{1}$, Sílvio C. de Oliveira ${ }^{2}$, Yana G. Ivanushko ${ }^{3}$, Viktoria $\mathbf{O}$. \\ Tkach ${ }^{4}$, Hanna Ya. Mytrofanova ${ }^{4}$, Anatolii O. Zadoia ${ }^{4}$, Petro I. Yagodynets ${ }^{1}$, Zholt O. Kormosh ${ }^{5}$ \\ 1 Chernivtsi National University, 58000, Kotsyubyns 'ky Str. 2, Chernivtsi, Ukraine \\ 2 Universidade Federal de Mato Grosso do Sul, Av. Sen. Felinto. Müller, 1555, C/P. 549, 79074-460, Campo Grande, MS, \\ Brazil \\ 3 Bukovinian State Medical University, 58001, Teatralna Sq., 9, Chernivtsi Ukraine \\ 4 Alfred Nobel University, 49000, Naberezhna Sicheslavska Str., 18, Dnipro, Ukraine \\ 5 Eastern European National University, 43000, Voli Ave., 13, Lutsk, Ukraine \\ * Correspondence: nightwatcher2401@gmail.com;
}

Received: 1.08.2020; Revised: 7.09.2020; Accepted: 8.09.2020; Published: 11.09.2020

\begin{abstract}
An interesting electro organic economic and green method of acetaldehyde to glyoxal conversion, based on electrochemical oxidation over a selenite-doped conducting polymer, has been described theoretically. A mathematical model, correspondent to the process, has been developed and analyzed by means of the linear stability theory and bifurcation analysis. The model analysis has confirmed the efficiency of the model for glyoxal laboratory and industrial production by the electrocatalytic method. Although the oscillatory behavior is possible in this system, it won't influence the glyoxal yield strongly.
\end{abstract}

Keywords: glyoxal; electroorganic synthesis; selenite-ion; conducting polymers; stable steady-state.

(C) 2020 by the authors. This article is an open-access article distributed under the terms and conditions of the Creative Commons Attribution (CC BY) license (https://creativecommons.org/licenses/by/4.0/).

\section{Introduction}

Glyoxal [1-4] is the simplest dialdehyde, frequently used by pharmaceutical and cosmetical industry for the production of drugs, food additives, lipsticks, chewing gums. Its formula is represented on Fig. 1:<smiles></smiles>

Figure 1. Glyoxal.

Glyoxal may be obtained in the industrial scale by different manners, including the electrooxidation of ethylene glycol [5], acetylene [6], and acetaldehyde, using highly specific oxidant - selenite-ion, according to the equation:

$$
\mathrm{CH}_{3} \mathrm{COH}+\mathrm{H}_{2} \mathrm{SeO}_{3} \rightarrow \mathrm{C}_{2} \mathrm{H}_{2} \mathrm{O}_{2}+\mathrm{Se}+2 \mathrm{H}_{2} \mathrm{O}
$$

All of these processes involve poisonous, expensive, and ecologically dangerous substances. The last process is used for glyoxal synthesis in the laboratory and in a low industrial scale. Its high-industrial use is impeded by both toxicity and expense of tetravalent selenium derivatives. This problem may be resolved by the development of the electrochemical analogs of the process, described by the reaction (1). In this process, the selenite-ion is hosted 
in carbon or conducting polymer matrix, as in [8-16], oxidizing acetaldehyde into glyoxal. Furtherly, it will be regenerated by the electrochemical reaction (2):

$$
\mathrm{Se}+3 \mathrm{H}_{2} \mathrm{O}-4 \mathrm{e}^{-} \rightarrow \mathrm{SeO}_{3}{ }^{2-}+6 \mathrm{H}^{+}
$$

Nevertheless, before its practical use, the electrocatalytic industrial process has to be analyzed from the theoretical point of view. This analysis not only provides the prevision about the behavior of the system and its efficiency but also verifies the possibility of the electrochemical instabilities, capable of the influencing strongly the system's behavior. These instabilities are typical for the oxidation of small organic molecules [17-18], including electropolymerization.

Another positive feature of the theoretical analysis is its possibility to compare the behavior of the described system with that of similar ones.

Therefore, the aim of this work is to analyze the acetaldehyde electro organic conversion into glyoxal, provided by a selenite-doped conducting polymer electrode. In order to achieve it, we realize the specific goals:

- $\quad$ suggestion of the mechanism of the reaction consequence, leading to the realization of the electro organic process;

- development of the balance equation mathematical model, correspondent to the electroanalytical system;

- $\quad$ analysis and interpretation of the model in terms of the electroanalytical use of the system;

- $\quad$ the seek for the possibility of electrochemical instabilities and for the factor, causing them;

- $\quad$ the comparison of the mentioned system's behavior with similar ones [19-21].

\section{System and its modeling}

Schematically, the electrocatalytic process, composed by the reactions $(1-2)$ may be exposed to the Fig. 2:

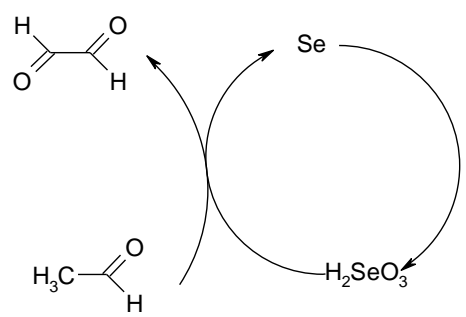

Figure 2. The scheme of acetaldehyde to glyoxal selenite-assisted electro organic conversion.

Therefore, in potentiostatic mode, the system's behavior will be described by an equation set of two variables:

a - acetaldehyde concentration in the pre-surface layer;

$\mathrm{s}$ - elementary selenium matrix coverage degree;

To simplify the modeling, we suppose that the reactor is intensively stirred, so we can neglect the convection flow. Also, we assume that the background electrolyte is in excess, so we can neglect the migration flow. The diffusion layer is supposed to be of a constant thickness, equal to $\delta$, and the concentration profile in it is supposed to be linear.

It is possible to show that the electroanalytical process will be described by the bivariant equation set, exposed as: 


$$
\left\{\begin{array}{c}
\frac{d a}{d t}=\frac{2}{\delta}\left(\frac{A}{\delta}\left(a_{0}-a\right)-r_{1}\right) \\
\frac{d s}{d t}=\frac{1}{S}\left(r_{1}-r_{2}\right)
\end{array}\right.
$$

Herein, A is the aldehyde diffusion coefficient, ao is its bulk concentration, $\mathrm{S}$ is the maximal selenium concentration, and the parameters $r$ stand for the correspondent reaction rates, capable of being calculated as:

$$
\begin{aligned}
& r_{1}=k_{1} a(1-s) \exp (\alpha s) \\
& r_{2}=k_{2} s \exp \left(\frac{4 F \varphi_{0}}{R T}\right)
\end{aligned}
$$

Herein, the parameters $\mathrm{k}$ are correspondent reaction rate constants, $\mathrm{F}$ is the Faraday number, $\alpha$ is the parameter describing the DEL influence of the ionic compound destruction on the chemical stage, $\varphi_{0}$ is the zero-charge potential related potencial slope, $\mathrm{R}$ is the universal gas constant and $\mathrm{T}$ is the reactor absolute temperature.

In this case, the system's behavior is presented as similar to those described in [1921], although somehow more dynamic. Therefore, the system to be described is an efficient system, as it will be confirmed below.

\section{Results and Discussion}

In order to describe the behavior of the system with the electrocatalytic conversion of acetaldehyde into glyoxal, assisted by the selenite-modified conducting polymer matrix, we analyze the equation-set (3), as also the algebraic relations ( $4-5)$, by means of linear stability theory. The steady-state Jacobian matrix members for this system may be described as:

$$
\left(\begin{array}{ll}
a_{11} & a_{12} \\
a_{21} & a_{22}
\end{array}\right)
$$

where:

$$
\begin{gathered}
a_{11}=\frac{2}{\delta}\left(-\frac{A}{\delta}-k_{1}(1-s) \exp (\alpha s)\right) \\
a_{12}=\frac{2}{\delta}\left(k_{1} a \exp (\alpha s)-\alpha k_{1} a(1-s) \exp (\alpha s)\right) \\
a_{21}=\frac{1}{s}\left(k_{1}(1-s) \exp (\alpha s)\right) \\
a_{22}=\frac{1}{s}\left(-k_{1} a \exp (\alpha s)+\alpha k_{1} a(1-s) \exp (\alpha s)-k_{2} \exp \left(\frac{4 F \varphi_{0}}{R T}\right)-\right. \\
\left.j k_{2} s \exp \left(\frac{4 F \varphi_{0}}{R T}\right)\right)
\end{gathered}
$$

The principal conditions for the system singular points are exposed on the Table 1.

Table 1. The bivariant systems main singular point requirements.

\begin{tabular}{c|c} 
Steady-state stability & $\operatorname{Tr} \mathrm{J}<0$, Det $\mathrm{J}>0$ \\
\hline Oscillatory instability (Hopf bifurcation) & $\operatorname{Tr} \mathrm{J}=0$, Det $\mathrm{J}>0$ \\
\hline Monotonic instability (saddle-node or static bifurcation) & $\operatorname{Tr} \mathrm{J}<0$, Det $\mathrm{J}=0$
\end{tabular}

Taking into account the elements (7) and (10), we may observe that in this case, the oscillatory behavior is somehow more probable than in the simplest systems [19]. The behavior of this system is very similar to that described in [20 - 21], as there is more than one factor influencing the double electric layer, comparing to the work [19].

The main condition of the oscillatory behavior $\operatorname{Tr} \mathrm{J}=0$ is satisfied if the main diagonal elements contain positive addendums, responsible to the positive callback. 
These elements are $\alpha k_{1} a(1-s) \exp (\alpha s)>0$, if $\alpha>0$ and $-j k_{2} s \exp \left(\frac{4 F \varphi_{0}}{R T}\right)<0$, if $\mathrm{j}<0$, and their positivity describes the periodic influence of the chemical and electrochemical stages. But if the element, analogous to $-j k_{2} s \exp \left(\frac{4 F \varphi_{0}}{R T}\right)$ is typical for all the similar systems $[19-21]$, the first one is characteristic only for the appearance, modification and disappearance of ionic compounds during the chemical stages, like those observed in [20-21]. The oscillations are expected to be frequent and of small amplitude.

In order to simplify the determinant analysis, we introduce new variables, describing the determinant as:

$$
\frac{2}{\delta G}\left|\begin{array}{cc}
-\kappa_{1}-\Xi & \Omega \\
\Xi & -\Omega-\Lambda
\end{array}\right|
$$

where

$$
\begin{gathered}
\kappa_{1}=\frac{s}{\delta} \\
\Xi=k_{1}(1-s) \exp (\alpha s) \\
\Omega=k_{1} a \exp (\alpha s)-\alpha k_{1} a(1-s) \exp (\alpha s) \\
\Lambda=k_{2} \exp \left(\frac{4 F \varphi_{0}}{R T}\right)+j k_{2} s \exp \left(\frac{4 F \varphi_{0}}{R T}\right)
\end{gathered}
$$

Mathematically, the oscillatory behavior conditions will be rewritten as:

$$
\left\{\begin{array}{c}
-\kappa_{1}-\Xi=\Lambda+\Omega \\
\kappa_{1} \Omega+\kappa_{1} \Lambda+\Xi \Lambda>0
\end{array}\right.
$$

We should admit that, if the influences of both factors are very strong, even if the first condition ( $\operatorname{Tr} J=0$ ) is satisfied, the second one won't be satisfied, as the determinant will possess negative values. Therefore, the oscillatory behavior in this system will be caused in this system with the influences of only one of the two mentioned DEL and will be realized only if the acetaldehyde diffusion is relatively slow.

The monotonic instability, which is manifested by N-shaped part of the voltammogram is also possible because of the mentioned influences. The condition of this instability will be described as:

$$
\left\{\begin{array}{c}
-\kappa_{1}-\Xi-\Omega-\Lambda<0 \\
\kappa_{1} \Omega=-\kappa_{1} \Lambda-\Xi \Lambda
\end{array}\right.
$$

And will only be satisfied if the parameters $\Lambda$, or $\Omega$ are negative, as only in this case, the second expression may have a null value.

It is possible to see that in the case of the positivity of the parameters $\Lambda$ and $\Omega$, describing the relative fragility of DEL influences, the stable steady-state will be warranted to be realized. Really, if the parameters $\Lambda$ and $\Omega$ are positive, the negativity of the matrix track will be warranted. Also, as the rest of the parameters are positive, the matrix determinant will be maintained positive.

Mathematically, the stability requisite will be described as: 


$$
\left\{\begin{array}{l}
-\kappa_{1}-\Xi-\Omega-\Lambda<0 \\
\kappa_{1} \Omega+\kappa_{1} \Lambda+\Xi \Lambda>0
\end{array}\right.
$$

And this requirement will be satisfied in a vast topological parameter zone. In other words, the stable steady-state is easy to maintain. This indicates an efficient and stable electrocatalytic process. The linear dependence between the acetaldehyde concentration in the electric current will be maintained firmly. The electrocatalytic process is diffusion-controlled.

This model is valid for potentiostatic mode. In galvanostatic mode, the potential is variable. Therefore, a third equation will be added to the equation-set (3). Also, the side reactions like selenide formation are also possible in this case, leading to the appearance of a more dynamic and somehow less efficient system. Therefore, the potentiostatic mode is more compatible and easier to realize from the economic point of view.

\section{Conclusions}

From the theoretical analysis of the acetaldehyde to glyoxal conversion for the pharmaceutical industry means, it is possible to conclude that: the electrocatalytic process of the acetaldehyde conversion into glyoxal is considered more advantageous than its chemical analog; - the system's behavior will be controlled by aldehyde diffusion. The stable steadystate will be formed easily and maintain simply; - the oxidized selenium form (selenite) is easily regenerated on the electrochemical stage, reducing the use of toxic and expensive selenium compounds; - the oscillatory behavior may be realized in the system, caused by DEL influences of both chemical and electrochemical stages. They are more capable of being realized if the aldehyde diffusion is slower;- from the economic point of view, the potentiostatic mode is more efficient due to the possibility of side reactions and rapid and great potential changes during the galvanostatic and potentiodynamic conversion.

\section{Funding}

This research received no external funding.

\section{Acknowledgment}

This research has no acknowledgment.

\section{Conflicts of Interest}

The authors declare no conflict of interest.

\section{References}

1. Vineeth, S.K.; Gadhave, R.V.; Gadekar, P.T. Glyoxal cross-linked polyvynil-alcohol-microcrystalline cellulose blend as wood adhesive with enhanced mechanical, termal and performance properties. Mat. Int. 2020, 2, 277-285.

2. Abdulwahed, M.; Mamoly, L.; Bosnali, W. A simple spectrophotometric method for determination of glyoxylic acid in its synthesis mixture. Int. J. Anal. Chem. 2020, 2020, https://doi.org/10.1155/2020/5417549.

3. Richter, K.N.; Revelo, N.H.; Seitz, K.J.; Helm, M.S.; Sarkar, D.; Saleeb, R.S.; 'D’Este, E.; Eberle, J.; Wagner, E.; Vogl, C.; Lazaro, D.F.; Richter, F.; Coy-Vergara, J.; Coceano, G.; Boyden, E.S.; Duncan, R.R.; Hell, S.W.; Lauterbach, M.A.; Lehnart, S.E.; Moser, T.; Outeiro, T.F.; Rehling, P.; Schwappach, B.; Testa, I.; Zapiec, B.; Rizzoli, S.O. Glyoxal as an alternative fixative to formaldehyde in immunostaining and superresolution microscopy. The EMBO J. 2018, 37, 139-159, https://doi.org/10.15252/embj.201695709. 
4. Çatak, J. Quantitative analysis of Glyoxal and Methylglyoxal Compounds in French Fry Samples by HPLC by HPLC Using 4-Nitro-1,2-Phenylenediamine as a Derivatizing Reagent Inn. Res. Rev. 2020, 4, $20-24$.

5. Zhang, Z.; Wang, Y.; Gu, J.; Lu, X.; Liao, X.; Shi, J.; Kim, C.; Lye, G.; Baganz, F.; Hao, J. Ethylene Glycol and Glycolic acid Production from Xylonic Acide by Enterobacter Cloacae, Microb. Cell. Fact. 2020, 19, https://doi.org/10.21203/rs.2.19479/v1.

6. Zhang, F.; Xiaofei, Yu; Chen, J.; Zhu, Z.; Yu, X.Y. Dark air-liquid interfacial chemistry of glyoxal and hydrogen peroxide, Clim. Atm. Sci, 2020, 2, https://doi.org/10.1038/s41612-019-0085-5.

7. Riley, H.L; Morley, J.F.; Child Friend, N.A. Selenium Dioxide, a New Oxidizing Agents. Part I. Its Reaction with Aldehydes and Ketones. J. Chem. Soc. 1932, 1875-1883, https://doi.org/10.1039/JR9320001875.

8. Kumar, Y.; Vashistha, V.K.; Das, D.K. Synthesis of Perovskite-type $\mathrm{NdFeO}_{3}$ nanoparticles and used as electrochemical sensor for detection of paracetamol. Lett. Appl. Nanobiosci. 2020, 9, 866-869, https://doi.org/10.33263/LIANBS91.866869.

9. Long, X. Electrochemical Sensors with Antifouling Properties for Sensitive Detection of Isoproturon Based on Glassy Carbon Electrode Modified with Nafion Membrane. Int. J. Electrochem. Sci. 2020, 15, 49644977, https://doi.org/10.20964/2020.06.58.

10. Sant'Anna, M.V.S.; Carvalho, S.W.M.M.; Gevaerd, A.; Silva, J.O.S.; Santos, E.; Carregosa, I.S.C.; Wisniewski, A.; Marcolino-Junior, L.H.; Bergamini, M.F.; Sussuchi, E.M. Electrochemical sensor based on biochar and reduced graphene oxide nanocomposite for carbendazim determination. Talanta 2020, 220, https://doi.org/10.1016/j.talanta.2020.121334

11. Laghrib, F.; Bakasse, M.; Lahrich, S.; El Mhammedi, M.A. Electrochemical sensors for improved detection of paraquat in food samples: A review. Materials Science and Engineering: C 2020, 107, https://doi.org/10.1016/j.msec.2019.110349.

12. Pérez-Fernández, B.; Costa-García, A.; de la Escosura-Muñiz, A.; Electrochemical (Bio)Sensors for Pesticide Detection Using Screen-Printed Electrodes. Biosensors 2020, 10, https://doi.org/10.3390/bios10040032.

13. Xiang, H.; Cai, Q.; Li, Y.; Zhang, Z.; Cao, L.; Li, K.; Yang, H. Sensors Applied for the Detection of Pesticides and Heavy Metals in Freshwaters. Journal of Sensors 2020, 2020, https://doi.org/10.1155/2020/8503491.

14. Noori, J.S.; Mortensen, J.; Geto, A. Recent Development on the Electrochemical Detection of Selected Pesticides: A Focused Review. Sensors 2020, 20, https://doi.org/10.3390/s20082221.

15. Hosny, N.M.; Sherif, Y. Molecular Docking Study on Some Isonicotinoyl Hidrazide Derivatives as Potencial Inhibitors of COVID-19. Lett. Appl. NanoBioSci. 2020, 9, 1217-1224, https://doi.org/10.33263/LIANBS93.12171224.

16. Lima, E.; Boto, R.; Ferreira, D.; R. Fernandes, J.; Almeida, P.; Ferreira, L.; Souto, E.B.; Silva, A.M.; Reis, L. Quinoline- and Benzoselenazole-Derived Unsymmetrical Squaraine Cyanine Dyes: Design, Synthesis, Photophysicochemical Features and Light-Triggerable Antiproliferative Effects against Breast Cancer Cell Lines. Materials 2020, 13, https://doi.org/10.3390/ma13112646.

17. Das, I.; Goel, N.; Agrawal, N.R.; Gupta, Growth patterns of dendrimers and electric potential oscillations during electropolymerization of pyrrole using mono-and mixed surfactants. J. Phys. Chem. B 2010, 114, 12888-12896, https://doi.org/10.1021/jp105183q.

18. Das, I.; Goel, N.; Gupta, S.K.; Agrawal, N.R. Electropolymerization of pyrrole: Dendrimers, nano-sized patterns and oscillations in potential in presence of aromatic and aliphatic surfactants. J. Electroanal. Chem. 2012, 670, 1-10, https://doi.org/10.1016/j.jelechem.2012.01.023.

19. Tkach, V.V.; Kushnir, M.V.; Ivanushko, Y.G.; de Oliviera, S. The Theoretical Description for Neotame Electrochemical Determination, Assisted by Vanadium Oxyhydroxide Composite with a Squarainic Dye. Appl. J. Env. Eng. Sci. 2020, 6, 109-115.

20. Tkach, V.V.; Kushnir, M.V.; Storoshchuk, N.M.; Ivanushko, Y. The Theoretical Description for the Confection of the Novel Thiourea-Based Active Surface for Cathodic Conducting Polymer Deposition. Appl. J. Env. Eng. Sci. 2020, 6, 143-148.

21. Tkach, V.; Kushnir, M.; Ivanushko, Y.; de Oliveira, S.; Reis, L.; Yagodynets', P.; Kormosh, Z. The Theoretical Description for the Electrochemical Synthesis of Squaraine Dye Doped Conducting Polymer. Appl. J. Env. Eng. Sci. 2020, 6, 51-56. 